\title{
"Antimicrobial activity of rhizospheric bacteria of Curcuma longa (Turmeric) producing metabolites against human pathogens"
}

\author{
Shraddha Dilip Mandale ${ }^{1}$, Vikas Dagar ${ }^{2}$, Vijay Dagar ${ }^{1}$ \\ ${ }^{I}$ (MUHS nashik, India) \\ ${ }^{2}$ (Assistant professor, MUHS Nashik, India)
}

\begin{abstract}
The present study was carried out to explore the production of antibiotics from soil microbes of medicinal Plant Curcuma longa (Turmeric). Soil samples of the Curcuma longa rhizosphere regions were collected from the different region in Akola City, Western Vidharbh region of Maharashtra. The bacterial culture from the soil samples were collected by the serial dilution and spread plate technique. The total 16 culture have been isolated from the soil samples and out of total 16 only 3 have been characterized which are potent isolates. These 3 isolates are active against the selected pathogens, E. coli, P. aeruginosa and S.aureus. These samples were labelled as S1,S2 and S3. The characterized 3 cultures were identified as Bacillus megatherium, Pseudomonas fluroscenes and Globicitella sulfidifacein by using Bergey's manual of systemic bacteriology. The Rhizospheric bacterial crude extract of Bacillus megatherium, Pseudomonas fluorescence, Globicatella sulfidifaciens were found to be more or less active against almost all tested pathogenic strains. Hence Curcuma longa can be employed as source of natural antimicrobials that can serve as an alternative to conventional medicines.It was concluded that the best activity have been shown by the Curcuma longa rhizospheric isolates (S2) which is of Pseudomonas fluorescens against all three human pathogenic organisms (E.coli, S.aureus, P.aeroginosa). The activity of rhizospheric isolates was showing best results against $S$. aureus
\end{abstract}

Keywords: Antimicrobial, rhizospheric and curcuma longa

\section{Introduction}

The world health organization (WHO) has listed 21000 plants which are used for medicinal purposes around the world. Among this 2500 species are in India, out of which 150 species are use commercially on a fairly, large scale, India is the largest producer of medicinal herbs and is called as botanical garden of the world. Plants are primary source of medicine, among the plants known for their medicinal values ${ }^{[1]}$ Rhizosphere is a narrow zone around a plant root. It is a dynamic region where various biological and chemical processes take place along with a variety of chemical release by roots and mediated by the soil microbes ${ }^{[2]}$. The rhizosphere is a densely populated area in which the roots must compete with the invading root systems of neighboring plant species for space, water, and mineral nutrients, and with soil-borne microorganisms, including bacteria, fungi, and insects feeding on an abundant source of organic material. Thus, root-root, root-microbe, and root-insect communications are likely continuous occurrences in this biologically active soil zone, but due to the underground nature of roots, these intriguing interactions have largely been overlooked ${ }^{[3]}$.

Given the complexity and biodiversity of the underground world, roots are clearly not passive targets for soil organisms. Rather, the compounds secreted by plant roots serve important roles as chemical attractants and repellents in the rhizosphere. The chemicals secreted into the soil by roots are broadly referred to as root exudates. Through the exudation of a wide variety of compounds, roots may regulate the soil microbial community in their immediate vicinity, cope with herbivores, encourage beneficial symbioses, change the chemical and physical properties of the soil, and inhibit the growth of competing plant species. Medicinal plants are considered to be very rich sources of metabolites. The plant chemicals are classified as primary and secondary metabolites. In higher plants such compounds offer concentration in seeds and vegetative storage organs and are needed for physiological development because of their role in basic cell metabolites. Primary metabolites obtain from higher plants for commercial use is high volume- low value bulk chemical ${ }^{[4]}$ (e.g. vegetable oil, fatty acid, carbohydrates etc).Secondary metabolites are classically organic compounds produce from micro-organism during the alteration of primary metabolites synthesis.Many among secondary metabolites have ecological function; which include defense mechanism also function as antimicrobial agents or antibiotics and by producing various pigments. Antibiotics are one of the most important and wide employed secondary metabolites produce by bacteria. The soil microbes are a major source of antibiotics various bacterial strains are selected for antibiotics production as its isolation, maintenance and strain improvements is easy. ${ }^{[3}$ Medicinal plants are considered to be very rich in secondary metabolites. Among them turmeric genus Curcuma belonging to the family Lamiaceae are very important for their therapeutic potentials Turmeric native in south east India has wide range of essential application like food and pharmaceutical products, cosmetics and textile industries. 
As agriculture and pharmaceutical are concerned it is indeed necessary to focus on plant growth, rhizosphere micro-organism and soil health. Certain metabolic pathways in the plant are induce by the infection with microorganisms and can satisfy the nutritional requirement of the plant. Major activity of the chemical constituents include significant antibacterial, anti-inflammatory, antioxidant, antipyretic, antiseptic, antifungal, anticancerous capabilities.A bioactive component Curcumin present in turmeric is responsible for wide spectrum of medicinal properties and have commercial applications. Curcumin helps in reducing cholesterol and also as a blood purifier. Phytochemicals in turmeric have been investigated in preliminary research for their potential effects on diseases such as cancer, arthritis, diabetes and other clinical disorders. Turmeric improves the action of liver and is traditional remedy jaundice in both ayurvedic medicines. ${ }^{[5]}$

\section{Material And Methods}

The present study is carried out by the antibacterial activity of rhizospheric bacteria of Curcuma longa producing metabolites against human bacterial pathogens. The bacterial culture from the soil samples were collected by the serial dilution and spread plate technique. The total 16 culture have been isolated from the soil samples and out of total 16 only 3 have been characterized which are potent isolates and labeled as S1,S2 and S3.Followed by various biochemical and microscopic test for identification of microrganisms.

\section{Sample Collection-}

Soil samples were collected from rhizospheric region of Curcuma longa plant located in different region in Akola city western Vidharbh region of Maharashtra. Total 16 rhizospheric soil samples were collected in the sterilized polythene bag containing soil sample were transfer immediately to laboratory. The collected Rhizospheric soil sample of Curcuma longa were weight $1 \mathrm{gm}$ aseptically and immediately transfer to $9 \mathrm{ml}$ saline suspension that is called Stock culture.

Step 1- Serial Dilution: Isolation of microbes from soil by serial dilution:Microbes are generally found in soil as mixed populations. The serial dilution is a method followed to get reduced number of colonies or less populated colonies from the sample. The microbes are successfully cultivable only in liquid media

and are generally isolated by serial dilution method ${ }^{[6]}$.After the rhizospheric soil was added to prepare stock solution further Serial dilution method was performed to get reduce number of bacteria. Dilution were made up to $10^{-8}$ to reduce the load of bacteria for better isolation of colonies.

\section{Procedure}

1. 10 test tubes were taken \& washed properly and 5 Petri dishes and 5 pipettes were taken (sterilized).

2. $100 \mathrm{ml}$ Nutrient agar was prepared (sterilized).

3. All the required materials including spreader, distilled water were autoclaved at $121^{0} \mathrm{C}$ for $15 \mathrm{lbs}$.

4. After sterilization antifungal tablet were added in Nutrient agar in powder form.

5. Nutrient agar was poured in sterilized petri plates and allowed to cool.

6. $1 \mathrm{gm}$. rhizospheric soil sample was weighted and added to $10 \mathrm{ml}$ saline sample in test tube. This is called stock solution.

7. $1 \mathrm{ml}$ stock solution was added into first test tube i.e. $10^{-1}$ and this serially diluted upto $10^{-8}$.

8. From serially diluted test tubes $10^{-5}, 10^{-6}, 10^{-7}, 10^{-8} 1 \mathrm{ml}$ of sample was transferred to solidified Nutrient agar plates.

9. Petri plates were labelled as per diluted $\left(10^{-5}, 10^{-6}, 10^{-7}, 10^{-8}\right)$

10. Spread the sample uniformly throughout the agar plates with the help of sterilized spreader.Inoculated agar plate was incubated at $37^{\circ} \mathrm{C}$ for $24 \mathrm{hrs}$

\section{Step 2-}

1. After incubation at $37^{\circ} \mathrm{C}$ for $24 \mathrm{hrs}$. Different colonies were observed on Nutrient agar plates.

2. Different Selective media were Prepared and autoclaved at $121^{\circ} \mathrm{C}$ for $15 \mathrm{lbs}$.

3. Single type of Loop full colonies were streaked on selective media by streak plate method.

4. Each Petri plate was labelled per selective media.

5. All the Petri plates were incubated in incubator at $37^{\circ} \mathrm{C}$ for $24 \mathrm{hrs}$.[inverted position]

Step 3-

1. After incubation period different types of colonies were observed on selective media.

2. Colony characteristic were observed and noted.

3. Single colony was streak on nutrient agar slant for the isolation of pure culture.

Step 4 - Isolation of crude extract producing antimicrobial substances. ${ }^{[7]}$

For the isolation of antimicrobial crude extract the test bacterial sample was inoculated in nutrient broth fermentation medium \& incubated at $37^{\circ} \mathrm{c}$ for $48 \mathrm{hrs}$. The fermented broth was then treated to separate the biomass from broth. The broth was then centrifuged at $4000 \mathrm{rpm}$ on for 15 minutes and then subjected to extraction with ethyl acetate by solvent extraction procedure equal volume of ethyl acetate was added to the filtrate and mixed well by vigorous shaking for 10 minutes. Tubes were allowed to settle for 5 minutes till two clear immiscible layers are formed. The upper layer containing the extracted compounds was separated and 
collected in another tube. This filtrate extract was evaporated to dryness in hot air oven. The extract residue was dissolved in dimethyl sulfoxide (DMSO) and stored at $4^{\circ} \mathrm{c}$ to be used as stock solution for antimicrobial assay.

Step 5-Antibiogram test ${ }^{[8]}$ : Bergey's manual of systemic bacteriology. Ninth edition john G. holt, Noer R. KreigPeter H A Sneath, James T. Staley_Nutrient agar plate were prepared. Spray $20 \mu$ of selected test pathogens (Staphylococcus aureus, Pseudomonas aeruginosa, Escherichia coli) were spread on to the solidified nutrient agar plates. Three wells were made at appropriate distance onto the agar plate with the help of gel puncture and filled using different concentration like $25 \mu \mathrm{l}, 50 \mu \mathrm{l}$, and $75 \mu \mathrm{l}$ of the bacterial isolates' broth extracts obtained from different strains. Petri plates were incubated at $37^{\circ} \mathrm{C}$ for overnight. Then the diameter of the zone of inhibition was measured in $\mathrm{mm}$ and noted. The antimicrobial activity was determined by measuring the clear zone around the wells.

Step 6-Staining :- staining was done by using methylene blue dye or basic fuschin and gram's staimimg for differentiation of microorganism as gram positive(purple colour) or negative(pink colour)

II) Motility Motility was studied for all the isolates. Nutrient broth was inoculated with the isolated with incubated at $37^{\circ} \mathrm{c}$ for 24 hours. After incubation, hanging drop preparation method was used to study the motility of the isolates.

III) Endospore test: An aqueous primary stain (malachite green) is applied and steamed to enhance penetration of the impermeable spore coats. Once stained the endospores do not readily decolorize and appear green within red cells. A thin smear of culture was made and Malachite green was added in the pressure of water vapours for 5 min. After washing Safranin was added to it for $40 \mathrm{sec}$ further it was again washed with distilled water, air dried and observed under the microscope.

IV) Acid fast staining: Smear is prepared on a glass slide and fixed by heat. The smear is flooded with carbolfuchsin and is heated up to steaming. Washed then slide with water. Decolourise the film by $20 \%$ sulphuric acid or mixture of acid and alcohol (conc. of HCL $3 \mathrm{ml}$ and $95 \%$ alcohol $97 \mathrm{ml}$ ). Tilled film is only faintly pink. The slide is again washed with water. Film was counter stained with metylene blue.

Step 7-Biochemical Test:The identification of bacteria is a systemic process that uses much different technology to narrow down the types of bacteria that are present in an unknown bacteria culture. It produces benefits for many aspect of research of microorganism and help physicians correctly treat patient. Multiple tests were performed to provide the fermentation abilities, presence of certain enzymes and certain biochemical test. Various tests involved in the identification of unknown bacteria. The standard biochemical tests were performed on the various isolates. These biochemical test were as-

I) Sugar Fermentation-Glucose, Lactose, Mannitol, Galactose, Mannose, Maltose, Arabinose, Ribose, Fructose, Raffinose, Sorbitol, Sucrose and Xylose fermentation broth media were inoculated with the isolates and incubated at $37^{\circ} \mathrm{C}$ for 24 hours. After incubation, tubes were observed for the acid and gas production.

Glucose- If acid is produced indicated by colour change and gas indicated from gas production in Durham's tubes test is positive test.

Lactose - If colour change will not occurs from blue to yellow and gas is not produced in Durham's tubes test is negative.

Mannitol-If is also same like lactose and maltose colour change indicates positive test and no colour change indicates negative test.

II) IMViC test

In biochemical test various test are performed they are.Indole test, methyl red, Vogas proskauer test and citrate test collectively known as IMViC test.

III) Enzymes test :- to classify Catalase, Oxidase, Urease, $\mathrm{NO}_{3}$ Reduction, $\mathrm{H}_{2} \mathrm{~S}$ Production, Starch hydrolysis -etc

\section{Observations}

Soil samples of the Curcuma longa rhizosphere regions were collected from the different region in Akola City, Western Vidharbh region of Maharashtra. The bacterial culture from the soil samples were collected by the serial dilution and spread plate technique. The total 16 culture have been isolated from the soil samples and out of total 16 only 3 have been characterized which are potent isolates. These 3 isolates are active against the selected pathogens, E. coli, P. aeruginosa and S.aureus. These samples were labelled as S1,S2 and S3. The characterized 3 cultures were Bacillus megatherium, Pseudomonas fluroscenes and Globicitella sulfidifacein. Table 1 shows Morphological characteristics of the isolated culture from the Curcuma longa rhizosperic soil sample.On the basis of cultural, Morphological and Biochemical characteristics. The potent isolates were identified by using Bergey's manual of systematic Bacteriology. According to this, gram's staining, catalase test, endospore test, acid fast staining, glucose fermentation test, mannitol fermentation test, lactose fermentation test, citrate utilization test, oxidase test, glucose oxidation test, and nitrate reduction test have been performed. Table 2 shows Morphological \& Biochemical Characteristics Further, the isolates have been tested for the activity to inhibit the growth of the selected human pathogens by antibiogram test. 
As shown in fig 1, There are two potent isolates have been found, which has shown the best activity against selected pathogens. Those isolates are S1 (B.megatherium) has shown zone of the inhibition $16.5 \mathrm{~mm}$ against $E$. coli and S3 (G.sulfidifaceins) has shown zone of the inhibition $16 \mathrm{~mm}$ against S.aureus. The S2 is the much more potent culture in comparison to other one. This culture has shown the best result against $S$. aureus in contrast to other pathogens. Between both the potent isolates, the $\mathrm{S} 2$ culture has the maximum activity against all the selected pathogens. This has been observed by comparing all the isolates activity of Curcuma longa rhizospheric soil samples.

\section{Tables And Figures}

Table no. 1:-Morphological characteristics of the isolated culture from the Curcuma longa rhizosperic soil sample.

\begin{tabular}{|c|c|c|c|}
\hline Sr.no & Sample & Colony C & \\
\hline \multirow{6}{*}{1.} & \multirow{6}{*}{ S1 } & Shape & Circular \\
\hline & & Size & $<0.01 \mathrm{~mm}$ \\
\hline & & Colour & Off white \\
\hline & & Texture & Smooth \\
\hline & & Elevation & Flat \\
\hline & & Opacity & Opaque \\
\hline \multirow{6}{*}{2.} & \multirow{6}{*}{$\mathbf{S 2}$} & Shape & Circular \\
\hline & & Size & $0.1 \mathrm{~mm}$ \\
\hline & & Colour & Yellowish green \\
\hline & & Texture & Hard \\
\hline & & Elevation & Flat \\
\hline & & Opacity & Opaque \\
\hline \multirow{6}{*}{3.} & \multirow{6}{*}{ S3 } & Shape & Circular \\
\hline & & Size & $<0.1 \mathrm{~mm}$ \\
\hline & & Colour & Pale yellow \\
\hline & & Texture & Smooth \\
\hline & & Elevation & Flat \\
\hline & & Opacity & Opaque \\
\hline
\end{tabular}

* Abrrivations : S1-Sample No.1, S2-Sample No.2 and S3- Sample No.3

Table no 2:-Comparision of Antimicrobial activity of S1,S2 and S3 At different concentration

\begin{tabular}{|c|c|c|c|c|c|c|c|c|c|}
\hline \multirow[t]{3}{*}{ Test bacterial strains } & \multicolumn{9}{|c|}{ Concentration } \\
\hline & \multicolumn{3}{|c|}{$25 \mu 1$} & \multicolumn{3}{|l|}{$50 \mu 1$} & \multicolumn{3}{|l|}{$75 \mu 1$} \\
\hline & S1 & S2 & S3 & S1 & $\mathrm{S} 2$ & S3 & $\mathrm{S} 1$ & S2 & S3 \\
\hline E.coli & $8 \mathrm{~mm}$ & $12 \mathrm{~mm}$ & $12 \mathrm{~mm}$ & $15 \mathrm{~mm}$ & $14 \mathrm{~mm}$ & $14 \mathrm{~mm}$ & $16.5 \mathrm{~mm}$ & $15 \mathrm{~mm}$ & $15 \mathrm{~mm}$ \\
\hline S.aureus & $11 \mathrm{~mm}$ & $12 \mathrm{~mm}$ & $13 \mathrm{~mm}$ & $14 \mathrm{~mm}$ & $16 \mathrm{~mm}$ & $16 \mathrm{~mm}$ & $15 \mathrm{~mm}$ & $17 \mathrm{~mm}$ & $16 \mathrm{~mm}$ \\
\hline P.aeruginosa & $12 \mathrm{~mm}$ & $11 \mathrm{~mm}$ & $10 \mathrm{~mm}$ & $13 \mathrm{~mm}$ & $11 \mathrm{~mm}$ & $12 \mathrm{~mm}$ & $16 \mathrm{~mm}$ & $14 \mathrm{~mm}$ & $14 \mathrm{~mm}$ \\
\hline
\end{tabular}

Table no 3:- Morphological \& Biochemical Characteristics

\begin{tabular}{|c|c|c|c|c|c|c|c|c|c|c|c|c|c|c|c|c|c|}
\hline \multirow{2}{*}{ 商 } & \multirow{2}{*}{ 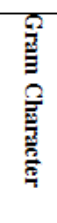 } & \multirow{2}{*}{ 朵 } & \multirow{2}{*}{ 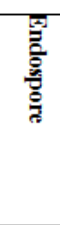 } & \multirow{2}{*}{ 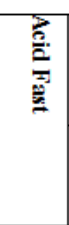 } & \multicolumn{3}{|c|}{$\begin{array}{l}\text { Sugar } \\
\text { fermentation }\end{array}$} & \multicolumn{4}{|c|}{ IMVIC } & \multicolumn{3}{|c|}{ Enzymes } & \multirow{2}{*}{ 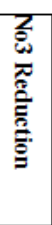 } & \multirow{2}{*}{ 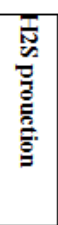 } & \multirow{2}{*}{ 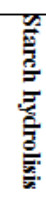 } \\
\hline & & & & & 产 & $\overline{\overline{\hat{a}}}$ & 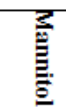 & $\overline{\bar{z}}$ & $\frac{3}{\pi}$ & $\leq$ & है & 啇 & 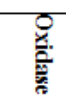 & 高 & & & \\
\hline S1 & + & + & + & -- & + & + & _ & - & -- & - & + & + & - & + & - & - & + \\
\hline S2 & & -- & -- & -- & + & & + & + & & & + & + & + & & .- & & + \\
\hline S3 & + & -- & -- & -- & + & _ & _ & -- & -- & _ & -- & -- & -- & _ & -- & + & + \\
\hline
\end{tabular}

Where, NM- Non motile , + Positive , - Negative , MR-Methyl red, VP- VogesProskaur

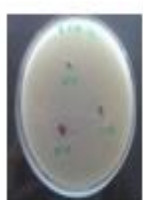

S1(a)

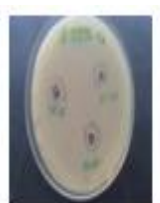

S1(b)

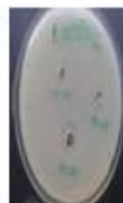

$\mathrm{S} 1(\mathrm{c})$

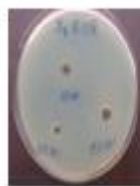

S2(a)

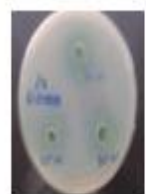

S2(b)

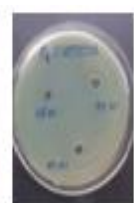

S2(c)

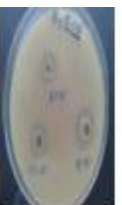

S3(a)

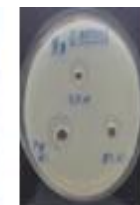

S3(b)

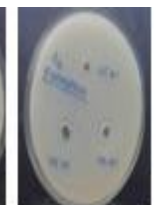

S3(c)

FIG 1 Antimicrobial activity of Sample S1 S2 and S3 against (a) E.coli (b) S aure and (c) p.aerogenosa at various concentration 


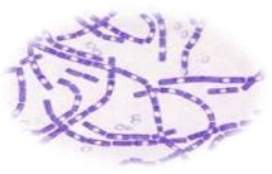

S1

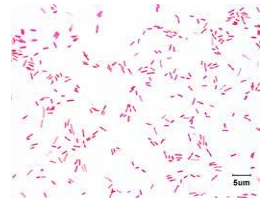

$\mathrm{S} 2$

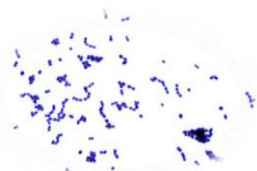

S3

Fig 2:- Microscopical characteristic of S1,S2 and S3 samples.S1 is Long rod shape, S2 is Short rod shape and $\mathrm{S} 3$ is Cocci shape

\section{Dicussion}

Roots serve many functions for a plant including anchorage and acquisition of vital nutrients and water necessary for growth. The plant root-soil interface is a dynamic region in which numerous biogeochemical processes take place driven by the physical activity, and the diversity of chemicals released by the plant root and mediated by soil microorganisms. In turn the processes occurring in this region control a host of reactions regulating terrestrial carbon and other element cycling that sustain plant growth and which have an enormous influence on plant and microbial community function and structure which greatly influence a variety of ecosystem level processes (van der Heijden et al., 2008; Wardle, 2004; Berg and Smalla, 2009). ${ }^{[9,10.11]}$ Pande A. Singh $^{[2]}$ worked on Comparative Study on Secondary Metabolites Producing Microbes Isolated from Rhizospheric \& Non-Rhizospheric Region of AzadirachtaIndicaand Oscimum tenuiflorum. Out of 18, only 8 were the potent isolates. The characterized 8 cultures were Sporosarcina, Streptococcus, Micrococcus luteus, Lactobacillus fermentum, Neisseria sicca, Escherichia coli and Streptococcus faecalis. Furthermore, the activity of the intracellular and extracellular secondary metabolites was observed and after various comparisons, it was concluded that the best activity have been shown by the Oscimum tenuiflorum non-rhizospheric isolate which is of Neisseria sicca against all three pathogens (S.aureus, P.aeruginosa and E.coli). The activity of the rhizospheric isolates was showing best result against $S$. aureus. The activity of the rhizospheric soil isolates may be affected by the plant root exudates.

Keshav Prasad Shukla et al., ${ }^{[12]}$ root exudates are one of the ways for plant communication to the neighboring plant and adjoining of microorganisms present in the rhizosphere of the root. The chemicals ingredients of the root exudates are specific to a particular plant species and also depend on the nearby biotic and abiotic environment. Root exudates mediate various positive and negative interactions like plant-plant and plantmicrobe interactions.

Surabhi Arora et al. ${ }^{[13]}$ studied by taking different Soil samples of Calotropis procera and Catharanthus roseus were subjected for antibacterial activity of microbes, extraction of secondary metabolites i.e both intracellular and extracellular, and characterization. Three type of colony were found i.e white, offwhite and yellow. Further using Bergey's manual Macrococcus luteus, N.sicca were obtained. Secondary metabolites i.e intracellular and extracellular was extracted using solvents chloroform and methanol. Antibiotic sensitivity test was performed against pathogen (E.coli, P.aeroginosa and S.aureus) and the best Zone of inhibition was of culture of Catharanthus roseusi.e $28.5 \mathrm{~mm}$. Different test were performed to characterize for medicinal purpose.Tanya Malviya, Amit Pandey ${ }^{[3]}$ Studied on Reduction of Antibiotics Isolated from Soil Bacteria from Rhizospheric and Non-Rhizospheric Region of Medicinal Plants Oscimum tenuiflorum and Aloe barbadensis. They were characterized and identified by Bergey's manual and the eight distinct cultures obtained were - Proteus vulgaris, Streptococcus epidermis, Lactobacillus fermentum, Bacillus cereus, N.mucosa, Sterptococcus equisimilis, Streptococcus faecalis and Bacillus subtilis. These cultures were further subjected to growth kinetic studies followed by optimized media production for each culture. It further led to extraction of intracellular and extracellular metabolites by solvent extraction method by methanol and ethyl acetate respectively. The metabolites were tested for their antimicrobial activity with the pathogens E.coli, S.aureus, P.aeruginosa, B.subtilis and S.typhi. The positive results obtained from AST of metabolites led to the determination of antibiotics in the metabolites from cultures by Chromatographic techniques by Thin Layer Chromatography.

In the present study, medicinal plant Curcuma longa has been selected, the rhizospheric region have been targeted to take the soil sample. The rhizosphere is the region adjacent to the plant root. The root exudates and the secondary metabolites secreted by the micro flora of the soil may affect each other and also to the plant health. There are total 18 cultures were isolated from these soil samples in which only 3 cultures have been screened. These 3 isolates are active against the selected pathogens, E. coli, P. aeruginosa and S.aureus. The characterized 3 cultures were Bacillus megatherium, Pseudomonas fluroscenes and Globicitella sulfidifacein. To characterize these cultures, Bergey's manual has been followed. According to this, gram's staining, catalase test, endospore test, acid fast staining, glucose fermentation test, mannitol fermentation test, lactose fermentation test, citrate utilization test, oxidase test, glucose oxidation test, and nitrate reduction test have been performed. The analysis of antibiogram of the entire characterized isolates has been observed before 
identification against selected pathogens. This has shown the drastic change in the activity of the isolates. The potent isolates were found able to produce metabolites on the basis of their specificity and hence the metabolites have shown the many fold increment in the activity of the isolates. Further, the isolates have been tested for the activity to inhibit the growth of the selected human pathogens by antibiogram test. There are two potent isolates have been found, which has shown the best activity against selected pathogens. Those isolates are S1 (B.megatherium) has shown zone of the inhibition $16.5 \mathrm{~mm}$ against E. coli and S3 (G.sulfidifaceins) has shown zone of the inhibition $16 \mathrm{~mm}$ against S.aureus. The S2 is the much more potent culture in comparison to other one. This culture has shown the best result against $S$. aureus in contrast to other pathogens. Between both the potent isolates, the S2 culture has the maximum activity against all the selected pathogens. This has been observed by comparing all the isolates activity of Curcuma longa rhizospheric soil samples.

\section{Conclusion}

The present study was an attempt to identify and pick out the versatile bacterial strains that display antimicrobial activity against variety of microbial pathogens intrinsically. Total 16 cultures were isolated from rhizospheric region of curcuma longa out of 3 were potent isolates characterized as Bacillus megatherium, Pseudomonas fluorescence, Globicatella sulfidifaciens. The Rhizosperic bacterial crude extract of Bacillus megatherium, Pseudomonas fluorescence, Globicatella sulfidifaciens were found to be more or less active against almost all tested pathogenic strains. Hence Curcuma longa can be employed as source of natural antimicrobials that can serve as an alternative to conventional medicines.It was concluded that the best activity have been shown by the Curcuma longa rhizospheric isolates (S2) which is of Pseudomonas fluorescens against all three human pathogenic organisms (E.coli, S.aureus, P.aeroginosa). The activity of rhizospheric isolates was showing best results against $S$. aureus The result of this study strongly supports that the bacterial isolates produces metabolites and may be used in the management of microbial infection and the present findings highlights the important for further investigation towards the goal of obtaining novel antimicrobial agent. The recovery of strains with antimicrobial activity suggest that the soil represents an ecological niche which harbours a largely uncharacterised microbial diversity and yet unexploited potential in the search for new metabolite.

\section{References}

[1]. Harikrishna Yadav Nanganuru (2013) : Effect Of Concentration Of Ocimumsactum Linn (Tulsi) Leaves Extract On The AAmylase, A- Glocusidase Activity And Microorganism Growth. International Journal Of Engineering Sciences \& Research Technology,2(2) : 2277-9655.

[2]. Amit pandey and Ankita Singh. (2013): A comparative study on secondary metabolites producing microbes isolated from rhizospheric and non rhizospheric region of Azadirachta indica and Oscimum tenuiflorum. International Journal of Pharmaceutical Research and Allied Science, 2(1):36-48.

[3]. Amit pande and Tanya Malviya (2014): Production of Antibiotics Isolated from Soil Bacteria from Rhizospheric and NonRhizospheric Region of Medicinal Plants. Indian journal of applied research, 4(8) :25-32

[4]. M. Wink, A.W. Alfermann, R. Franke et al(2005): Sustainable bioproduction of phytochemicals by plant in vitro cultures: anticancer agents.Plant Genet. Res., 3 (2005), pp. 90-100

[5]. Sahdeo Prasad and Bharat B. Aggarwal Turmeric, the Golden Spice From Traditional Medicine to Modern Medicine, Herbal Medicine: Biomolecular and Clinical Aspects. 2nd edition.2011

[6]. Singh .A and Mishra .S; Isolation and Biochemical Characterization of Antibiotic Producing Microorganism from Waste Soil Samples of Certain Industrial areas of India; IOSR-JPBS; 5(6): 2013; 80-

[7]. Hema Shenpagam .N, Kanchana Devi .D, Sinduja .G and Sandhya .R; Isolation of endophytic actinomycetes from medicinal plants and its mutational effect in biocontrol activity; IJPSR; 3(11): 2012; 4338-4344 |

[8]. Bergey's manual of systemic bacteriology. Ninth edition john G. holt, Noer R. KreigPeter H A Sneath, James T. Staley

[9]. van der Heijden MGA, Bardgett RD, Van Straalen NM (2008) The unseen majority: soil microbes as drivers of plant diversity and productivity in terrestrial ecosystems. Ecol Lett 11:296-310

[10]. Wardle DA ${ }^{1}$, Bardgett RD, Klironomos JN, et al:(2004). Ecological linkages between aboveground and belowground biota. Science. 2004 Jun 11;304(5677):1629-33.

[11]. Berg G, Smalla K (2009) Plant species and soil type cooperatively shape the structure and function of microbial communities in the rhizosphere. FEMS Microbiol Ecol 68:1-13

[12]. Keshav Prasad Shukla, Shivesh Sharma*, Nand Kumar Singh et al(2011) Nature and role of root exudates: Efficacy in bioremediation: African Journal of Biotechnology Vol. 10(48), pp. 9717-9724

[13]. Surabhi Arora ,Dipika Nandi ,Namrata Prasad est al (2013): Isolation and Characterisation of Antibiotic producing Microbes present in Rhizospheric soil. International Journal of Scientific \& Engineering Research, Volume 4(9):1157-1166 